\title{
CIRURGIA GASTROINTESTINAL NO TRATAMENTO DA DIABETE TIPO 2
}

\author{
Gastrointestinal surgery for the treatment of type 2 diabetes
}

\author{
Alexandre Coutinho Teixeira de FREITAS
}

ABCDDV/541

Freitas ACT. Cirurgia gastrointestinal no tratamento da diabete tipo 2. ABCD Arq Bras Cir Dig 2007; 20(2):119-26.

RESUMO - Racional - Evidências científicas demonstram o controle metabólico da diabete tipo 2 obtido com diversas intervenções sobre o sistema gastrointestinal, principalmente as operações bariátricas. Objetivos - Revisar os dados da literatura referentes aos efeitos da cirurgia gastrointestinal na diabete tipo 2, especialmente os relacionados ao controle metabólico e sua fisiopatologia. Metodos - Foi realizada pesquisa no Medline em páginas da internet procurando referências de artigos de maior relevância e estudos apresentados e publicados nos anais da conferência de Roma sobre os efeitos da cirurgia gastrointestinal no tratamento da diabete tipo 2 em 2007. Revisão da literatura - Entre as operações bariátricas, as derivações biliopancreáticas apresentam as mais altas taxas de controle da diabete tipo 2, seguidas pelo bypass gástrico e pela banda gástrica. Esse controle está relacionado à perda de peso e redução da ingestão de alimentos. As derivações biliopancreáticas e o bypass gástrico apresentam ainda efeito importante promovido por modificações hormonais. Os hormônios mais significativos são: o GLP-1, o GIP, o PYY, a grelina, a leptina, o IGF-1 e a adiponectina. Além dos efeitos sobre o controle do apetite no hipotálamo, os hormônios apresentam ação sobre as células $\beta$, promovem a secreção de insulina e diminuem sua resistência periférica. Duas hipóteses foram formuladas para explicar as modificações desses hormônios: a do intestino anterior, em que a exclusão do duodeno e do jejuno proximal previne a secreção de algum agente ainda não identificado que promove a resistência periférica à insulina; e a do intestino posterior, em que a derivação intestinal promove a passagem rápida do quimo até o intestino distal e induz à secreção precoce dos hormônios que promovem o controle da diabete. Além das operações bariátricas tradicionais, novos procedimentos promissores foram desenvolvidos nos últimos anos. Entre eles estão o bypass duodeno-jejunal, a interposição ileal e as ressecções intestinais associadas à gastrectomia vertical. Esses novos procedimentos ainda são considerados experimentais. Conclusão - Os procedimentos cirúrgicos sobre o trato gastrointestinal, principalmente as cirurgias bariátricas, proporcionam controle metabólico da diabete tipo 2. Entre as cirurgias bariátricas os melhores resultados são obtidos com as derivações biliopancreáticas seguidas pelo bypass gástrico e pela banda gástrica. Novos procedimentos cirúrgicos, ainda considerados experimentais, estão demonstrando resultados preliminares favoráveis ao controle metabólico da diabete tipo 2

DESCRITORES - Cirurgia bariátrica. Diabetes mellitus. Derivação biliopancreática. Derivação gástrica. Hormônios gastrointestinais.

\section{INTRODUÇÃO}

A diabete melito acomete cerca de 190 milhões de pessoas no mundo. Sua incidência está em franca evolução com projeção de acometer cerca de 324 milhões de pessoas em $2025^{62}$. A diabete tipo 2 representa $90 \%$ dos casos $^{71}$. Sua fisiopatologia ainda é controversa, mas muitos autores concordam em uma associação entre a resistência periférica a insulina e progressiva deterioração da função da célula $\beta$ nas ilhotas de Langerhans ${ }^{6}$. A obesidade, especialmente a visceral, é fator que leva à diminuição da sensibilidade à insulina e está associada à intolerância à glicose, dislipidemia e hipertensão arterial, todos integrantes da síndrome metabólica e considerados fatores de risco para o sistema cardiovascular. A íntima associação entre a diabete e a obesidade fez com que muitos autores de língua inglesa cunhassem o termo "diabesity".

A obesidade e co-morbidades relacionadas, entre elas a diabete tipo 2, aumentam a morbidade e a mortalidade da população acometida. Estudo com 457.785 pacientes mostrou relação direta entre o aumento da mortalidade

Trabalho realizado no Serviço de Cirurgia do Aparelho Digestivo do Hospital de Clínicas da Universidade Federal do Paraná, Curitiba, PR, Brasil.

Endereço para correspondência: Alexandre Coutinho Teixeira de Freitas, e-mail alexandrefreitas@ufpr.br e o aumento do índice de massa corporal ${ }^{5}$. No Brasil a obesidade apresentou incremento significativo nos últimos anos. Segundo dados da Pesquisa de Orçamentos Familiares do IBGE, nos anos de 1974 e 1975 a incidência de obesidade era de $2,8 \%$ para o sexo masculino e de $7,8 \%$ para o feminino ${ }^{30}$. Nos anos de 2002 e 2003 passou para $8,9 \%$ no sexo masculino e $13,1 \%$ no feminino.

O tratamento clínico da diabete tipo 2 é considerado ineficaz em um número significativo de casos. Nos últimos anos, apesar de modificações nas medicações utilizadas, dados da literatura mostram piora das taxas de controle metabólico da diabete. Segundo o estudo NHANES III (1988-1994), 44,5\% dos pacientes apresentaram níveis de hemoglobina glicosilada menor que $7 \%$ com o tratamento clínico; o estudo NHANES 1999-2000 demonstrou redução para $35,8 \%$ na proporção de casos com controle metabólico ${ }^{34}$. Muitos pacientes evoluem com complicações secundárias. A diabete é a principal causa de insuficiência renal crônica, cegueira e amputação de membros inferiores, também é considerada o principal fator de risco independente para doença coronariana ${ }^{45}$.

Nos últimos anos diversos estudos demonstraram a capacidade da cirurgia bariátrica promover controle da diabete e das outras co-morbidades associadas ${ }^{3,4,49,50,64}$. Existem também relatos de novas técnicas cirúrgicas 
promissoras.

Esse estudo tem por objetivo revisar os dados da literatura referentes aos efeitos da cirurgia gastrointestinal na diabete tipo 2, especialmente os relacionados ao controle metabólico e sua fisiopatologia.

\section{MÉTODO}

Foi realizada pesquisa no Medline com os termos "gastric bypass and diabetes mellitus, biliopancreatic diversion and diabetes mellitus, gastric banding and diabetes mellitus". Foram selecionados artigos de revisão, meta-análises, estudos randomizados controlados, estudos clínicos e protocolos em seres humanos e em animais, na língua inglesa publicados nos últimos 10 anos. Os trabalhos considerados de maior relevância foram analisados. Também foram analisadas páginas da internet relacionadas ao assunto e outras publicações selecionadas das referências bibliográficas dos artigos obtidos na pesquisa no Medline e considerados de maior interesse. Estudos apresentados e publicados nos anais da conferência de Roma sobre os efeitos da cirurgia gastrointestinal no tratamento da diabete tipo 2, em 2007 (International Conference on Gastrointestinal Surgery to Treat Type 2 Diabetes), também foram incluídos.

\section{REVISÃO DA LITERATURA}

\section{Resultados da cirurgia bariátrica na diabete tipo 2}

Buchwald et al. ${ }^{3}$ realizaram metanálise em 2004 demonstrando controle da diabete em $47,8 \%$ dos pacientes submetidos à banda gástrica, $83,6 \%$ dos submetidos a bypass gástrico e $97,9 \%$ dos com derivação biliopancreática. Os mesmos autores apresentaram dados de metanálise mais recente, com amplitude maior de estudos. Inicialmente foram incluídos 621 estudos com 135.246 pacientes. Destes, 16 abordaram diretamente a evolução da diabete. Foram incluídos 11.175 pacientes com IMC médio de 47,9 $\mathrm{kg} / \mathrm{m}^{2}$. Em termos gerais, os procedimentos cirúrgicos promoveram controle da diabete em $77 \%$ dos casos e em $84 \%$ foi observada melhora ou controle. A derivação biliopancreática promoveu controle da diabete em 95,1\% dos casos, o bypass gástrico em $80,3 \%$ e a banda gástrica ajustável em 53\%. Essa proporção de controle foi igual quando considerada a análise com menos de 2 anos e com mais de 2 anos de pós-operatório.

Estudo prospectivo e controlado realizado na Suécia (Swedish Obese Subjects Study Scientific Group) demonstrou os resultados da banda gástrica ajustável em 607 pacientes e do bypass gástrico em 34 pacientes no controle da diabete tipo 2 após período de seguimento de 10 anos $^{64}$. Após esse período, a perda de peso foi de $14,7 \pm 12 \%$ para os submetidos à banda gástrica ajustável e $25 \pm 11 \%$ para os com bypass gástrico. O grupo controle, com 627 pacientes, pareados um a um de acordo com 18 variáveis, apresentou ganho de peso de $1,6 \pm 12 \%$. Após 10 anos, a incidência de diabete foi maior no grupo controle (24\%) em relação ao grupo submetido à cirurgia bariátrica $(7 \%)$. Os operados apresentaram também menores taxas de dislipidemia e hiperuricemia.

Outro estudo demonstrou os resultados após período de 14 anos de seguimento ${ }^{50}$. A perda de excesso de peso foi de $49 \%$ em 608 pacientes submetidos ao bypass gástrico. Dos 146 portadores de diabete tipo 2, 121 (83\%) apresentaram controle da doença com níveis normais de glicemia de jejum, hemoglobina glicosilada e insulina sérica. Ainda, 150 dos 152 pacientes com intolerância a glicose tornaram-se normoglicêmicos.

\section{Mecanismos de resolução da diabete}

Diversos mecanismos contribuem para o controle da diabete no pós-operatório da cirurgia bariátrica. A explicação mais óbvia é que a perda de peso promove diminuição da resistência periférica à insulina ${ }^{10}$. Foi demonstrado que a induzida pelo bypass gástrico incrementa os níveis de adiponectina, uma adipocina que aumenta a sensibilidade à insulina ${ }^{23,29,48}$. Também foi demonstrado que incrementa a concentração de receptores para a insulina no músculo e reduz a quantidade de lipídios intramusculares e moléculas de acetil co-enzima $\mathrm{A}$, ambos indicativos de redução da resistência periférica à insulina ${ }^{23,29,48}$. O transporte de glicose para as fibras musculares é aumentado após o bypass gástrico e a disponibilidade de glicose corporal analisada pela técnica do clamp euglicêmico incrementa de $27 \%$ para $78 \%$ dos níveis observados em um grupo controle com peso normal ${ }^{19}$. Ainda, a medida da sensibilidade à insulina aumenta 4 a 5 vezes após a perda de peso ${ }^{29,48}$.

No entanto, a perda de peso isoladamente não é a única responsável pelo controle da diabete no pós-operatório. Estudos demonstram que os efeitos da cirurgia bariátrica sobre o metabolismo glicêmico ocorrem dias após a realização do procedimento, momento no qual ainda não foi observada grande perda de peso ${ }^{42,52,60}$. Morinigo et al..$^{42}$ analisaram o metabolismo glicêmico no pré-operatório imediato e no $6^{\circ}$ dia de pós-operatório em pacientes submetidos ao bypass gástrico. Os autores observaram redução da área abaixo da curva da insulina após o teste de tolerância à glicose no $6^{\circ}$ dia de pós-operatório em relação ao dia da operação. Essa melhora na resistência periférica à insulina também foi demonstrada através do método HOMA.

Outra explicação para o controle metabólico da diabete é a drástica redução na ingestão de alimentos observada no pós-operatório imediato, promovendo redução da necessidade de produção de insulina nas células $\beta$ e melhora do metabolismo glicêmico. Já são bem descritos os efeitos do jejum ou a drástica redução da ingestão sobre a diabete em diversas outras situações. Sem dúvida, esse mecanismo é importante no controle após a cirurgia bariátrica, no entanto não explica totalmente o fenômeno ${ }^{10}$. A comparação entre os resultados de operações puramente restritivas, como a banda gástrica, com operações mistas, como o bypass gástrico, indica outro mecanismo. As operações restritivas e as mistas apresentam o mesmo efeito sobre a capacidade de ingestão de alimentos no pós-operatório 
imediato, mas promovem controle da diabete em $50 \%$ e $84 \%$ dos casos, respectivamente ${ }^{3}$. Ainda, em contraste com o que ocorre nas operações mistas, nas restritivas esse controle ocorre tardiamente, após a ocorrência de perda de peso.

A análise do efeito sobre a diabete observado na derivação biliopancreática também indica outro mecanismo além da redução na ingestão e na perda de peso. Nesse procedimento, o componente restritivo é menos intenso e a ingestão alimentar é normalizada precocemente no pós-operatório. No entanto, estudos mostram controle da diabete após normalização da dieta em período no qual ainda não se observou redução significativa do peso ${ }^{1,61}$.

O terceiro mecanismo de controle da diabete no pósoperatório está relacionado com a secreção hormonal intestinal. Diversos hormônios, entre eles os que se incluem na categoria das incretinas, apresentam ação anti-apoptótica sobre as células $\beta$ nas ilhotas de Langerhans e estimulam a produção de insulina ${ }^{12}$. Rubino et al. ${ }^{55}$ realizaram estudo em ratos Goto-Karizaki diabéticos submetidos a bypass duodenojejunal com a finalidade de demonstrar a ação desses hormônios. Nesse procedimento, o estômago é deixado intacto, ou seja, sem o componente restritivo. Realiza-se somente secção ao nível do piloro e uma gastrojejunostomia em Y-de-Roux com derivação de parte do jejuno proximal de maneira semelhante à realizada nas operações de bypass gástrico. Os ratos dessa linhagem não são obesos e são considerados como modelo experimental de diabete tipo 2 . Em comparação com o grupo controle houve melhora do níveis de glicose de jejum e redução de mais de $40 \%$ na área abaixo da curva para glicose no teste de tolerância à glicose endovenosa. A melhora da diabete foi obtida sem mudanças no peso e na ingestão alimentar, dos animais em relação ao grupo controle. Os autores concluem que a derivação do duodeno e do jejuno proximal controla diretamente a diabete, independente de perda de peso ou tratamento da obesidade. Esses achados sugerem papel potencial do intestino proximal na patogênese da diabete tipo 2 .

Duas hipóteses foram propostas para explicar o mecanismo de ação da cirurgia bariátrica nos hormônios intestinais e na diabete: a hipótese do intestino anterior e a do intestino posterior ${ }^{54,55,56,57,10,12}$. Na primeira, a exclusão do duodeno e do jejuno proximal previne a secreção de algum agente promotor da resistência periférica à insulina $a^{24,51,54}$. Até o momento nenhuma molécula foi identificada. A hipótese do intestino posterior é a que apresenta maior suporte com dados da literatura. Nesse caso, a derivação intestinal promove a passagem rápida do quimo até o intestino distal e induz a secreção precoce de alguns hormônios que promoveriam melhora da diabete ${ }^{10,12,54,55,57}$. Estudos de interposição ileal em roedores suportam essa hipótese. Nesse procedimento, um segmento do íleo, com preservação de seu suprimento vascular e nervoso, é interposto ao jejuno proximal, aonde é exposto precocemente aos alimentos ingeridos. Alguns estudos mostram redução da ingestão alimentar, perda de peso, melhora da sensibilidade à insulina e, possivelmente, melhora na função das células $\beta^{32,65}$. Outros autores demonstraram melhora no metabo- lismo glicêmico na ausência de perda de peso e alteração da ingestão de alimentos ${ }^{47}$.

Ainda não está estabelecido qual dessas duas hipóteses é a responsável pelo controle da diabete após a cirurgia bariátrica e existe a possibilidade de que elas não sejam excludentes. Para tentar esclarecer essa questão, Rubino et al. ${ }^{57}$ realizaram outro estudo em ratos Goto-Karisaki submetidos ao bypass duodeno-jejunal, procedimento que permite a passagem rápida dos alimentos ingeridos para as porções distais do intestino associado à exclusão do duodeno e jejuno proximal. Outro grupo de animais foi submetido à gastroenteroanastomose látero-lateral. Este procedimento foi confeccionado com uma alça aferente com o mesmo comprimento da alça biliopancreática do bypass duodenojejunal e permite a passagem rápida dos alimentos ingeridos para as porções distais do intestino, porém não exclui o duodeno e jejuno proximal. Após 4 semanas esses dois grupos de animais foram reoperados: os com o bypass duodenojejunal foram submetidos à anastomose entre o piloro e o duodeno, restabelecendo o trânsito alimentar nas porções exclusas; os com gastroenteroanastomose foram submetidos à secção entre o piloro e o duodeno, tornado-os exclusos do trânsito alimentar. Os animais foram avaliados com teste de tolerância à glicose oral após a primeira e após a segunda operação. A ingestão de alimentos foi igual em ambos os grupos e em relação ao grupo controle. Após a primeira operação, o teste de tolerância a glicose via oral mostrou menor área abaixo da curva para glicose nos animais submetidos ao bypass duodenojejunal em relação aos submetidos à gastroenteroanastomose e aos do grupo controle. No entanto, após a segunda operação, os inicialmente submetidos à gastroenteroanastomose e depois à secção entre o piloro e o duodeno, apresentaram redução da área abaixo da curva para glicose. Os inicialmente submetidos ao bypass duodenojejunal apresentaram elevação da área abaixo da curva para glicose. Esses resultados demonstram o efeito negativo da passagem do trânsito alimentar pelo duodeno para o controle metabólico em ratos diabéticos. Os autores concluem que esses resultados suportam a hipótese do intestino anterior para o controle da diabete no pós-operatório de cirurgia bariátrica. Referem ainda que, na hipótese do intestino posterior tivesse sido dominante, esperariam que tanto o bypass duodeno-jejunal quanto a gastroenteroanastomose promovessem o controle da diabete, visto ambos os procedimentos permitirem a passagem precoce dos nutrientes para as porções distais do intestino.

\section{Efeito da cirurgia bariátrica nos hormônios relacio- nados com a diabete}

Os hormônios produzidos pelo trato gastrointestinal estão envolvidos no processo da digestão, da saciedade, da fome e do metabolismo dos carboidratos, dos lipídios e das proteínas. Esses hormônios, em conjunto com estímulos provenientes do sistema nervoso simpático e parasimpático, atuam ao nível do hipotálamo e regulam a secreção local de neuropeptídio Y, um peptídio orexigênico, e de serotonina 
e hormônio alfa-melanocítico, ambos anorexigênicos. O balanço entre eles determina as sensações de fome ou saciedade. Os hormônios do trato gastrointestinal também exercem efeito chamado de "freio ileal", reduzindo a motilidade da porção proximal do trato digestório. Esse efeito está intimamente relacionado com a taxa de absorção de glicose e outros elementos. Os hormônios apresentam ainda efeito direto ou indireto sobre a secreção e sobre a resistência periférica a insulina.

Esses hormônios são produzidos pela mucosa do estômago, do jejuno, do íleo, do cólon e pelo fígado ${ }^{17,31}$. Entre os hormônios com origem no íleo e no cólon são descritos: os produzidos pelas células L - GLP1 (glucagon like peptide 1), oxintomodulina e enteroglucagon; os secretados através de estímulos nervosos - peptídio YY e neurotensina ${ }^{12,37,43,42,67}$. Na mucosa do fundo gástrico ocorre a produção de grelina; na mucosa do duodeno e jejuno ocorre a produção de GIP (glucose-dependent insulinotropic peptide) e no fígado ocorre a produção de IGF-1 (insulin growth factor 1). Hormônios produzidos em outros locais além do trato gastrointestinal também apresentam efeito sobre o metabolismo da glicose. Entre eles estão a leptina, a adiponectina, o fator de necrose tumoral alfa, a interleucina 6 e a resistina, todos considerados como adipocinas produzidas pelo tecido adiposo.

Nos últimos anos, diversos estudos analisaram o efeito das cirurgias bariátricas sobre os hormônios do trato digestório. Os resultados forneceram informações não somente a respeito dos mecanismos de controle da diabete tipo 2 no pós-operatório, mas também a respeito da própria fisiopatologia da doença ainda não completamente esclarecida. Serão discutidas as alterações nos hormônios considerados mais relevantes.

O GLP-1 e o GIP são os mais próximos do que se considera como incretinas. As incretinas são descritas como uma classe de moléculas que promovem aumento da produção de insulina pelas células $\beta$ das ilhotas de Langerhans em resposta às refeições mesmo antes do aumento da glicemia. Também reduzem a absorção dos nutrientes através da diminuição do esvaziamento gástrico e diminuição do apetite. $\mathrm{O}$ termo incretina foi inicialmente proposto por La Barre em 1932 para descrever um hormônio extraído da mucosa intestinal que provocava hipoglicemia.

A hipótese do intestino posterior é uma das possíveis explicações para o controle metabólico da diabete após a realização de bypass gástrico ou derivações biliopancreáticas. A passagem rápida dos alimentos até o íleo e o cólon causa a secreção precoce de peptídios relacionados diretamente ou indiretamente com o metabolismo da glicose, principalmente através do estímulo para secreção de insulina. Diversos autores demonstraram a capacidade do bypass gástrico e das derivações biliopancreáticas elevarem os níveis séricos de GLP-1 $1^{12,37,43,42,67}$. Esse peptídio tem sido considerado como o principal responsável pelo controle precoce da diabete após o bypass gástrico. É produzido pela translocação do gene proglucagon no intestino em resposta à presença de nutrientes no seu lúmen. Entre as ações do GLP-1 observam-se redução do apetite, aumento da produção de insulina, indução da proliferação de células $\beta$, efeito anti-apoptótico nas células $\beta$, inibição da produção de glucagon e diminuição da resistência à insulina ${ }^{15,16,70}$. Atualmente, o uso clínico de agonistas do peptídio GLP-1 como o exenatide tem demonstrado efeitos positivos em relação ao controle metabólico da diabete ${ }^{12}$.

O GIP é um hormônio membro da família da secretina e é produzido pelas células $\mathrm{K}$ da mucosa do duodeno e jejuno em resposta a ingestão de glicose e gordura. Foi isolado e teve sua seqüência gênica determinada em 1970. Promove estímulo para a produção de insulina e tem efeito positivo sobre a proliferação de células $\beta$. Alguns estudos o citam como importante fator na fisiopatologia da diabete tipo 2 , visto ocorrer diminuição da expressão de seus receptores no pâncreas e conseqüente atenuação de seu efeito incretina (resistência ao GIP) ${ }^{38,46}$. Alguns autores especulam que, em indivíduos susceptíveis, a ingestão crônica de nutrientes ricos em glicose e gordura promove no duodeno e no jejuno proximal a produção de um fator desconhecido que reduz a expressão dos receptores para o GIP ${ }^{51,52,54,55,57}$. Como conseqüência, haveria diminuição da produção de insulina e diabete. Estudo demonstrou elevação de GIP em pacientes com diabete tipo 2 no pré-operatório de bypass gástrico, situação compatível com a resistência ao GIP $^{56}$. No pós-operatório ocorreu diminuição significativa de seu nível sérico. Os autores sugerem que a derivação do duodeno e jejuno proximal promoveria diminuição da produção desse fator desconhecido, melhora da resistência ao GIP e diminuição do seu nível sérico.

O IGF-1 é um peptídio produzido principalmente pelo fígado que apresenta estrutura molecular semelhante à da insulina. A administração in vivo de IGF-1 apresenta potente efeito hipoglicemiante e reduz com eficácia a glicemia em pacientes com diabete tipo 1 e tipo $2^{36,63}$. Níveis diminuídos de IGF-1 foram detectados em pacientes com diabete tipo $2^{68}$. O IGF-1 apresenta elevação de seus níveis séricos no pós-operatório de bypass gástrico e derivação biliopancreática ${ }^{25,53}$.

A grelina, produzida pelas células A das glândulas oxínticas no fundo gástrico, normalmente está elevada durante o jejum. Ao nível do hipotálamo ela promove a expressão do hormônio orexigênico neuropeptídio $\mathrm{Y}$ estimulando o apetite e a ingestão alimentar ${ }^{66}$. A distensão do fundo gástrico, obtida com a ingestão de alimentos, induz à diminuição da produção de grelina e proporciona um estímulo anorexigênico que determina a saciedade. Após o bypass gástrico, observa-se redução dos níveis séricos de grelina ${ }^{8,9,11,22,21}$. Estudo demonstrou redução dos níveis de grelina após o bypass gástrico para valor 77\% menor que o de um grupo controle pareado de pessoas magras e $72 \%$ menor que o de um grupo controle pareado de pessoas obesas ${ }^{8}$. Normalmente o estímulo para secreção ou não da grelina é cíclico, envolvendo períodos de jejum alternados com períodos pós-prandiais. A exclusão do fundo gástrico do trânsito não permite sua distensão após a ingestão alimentar. A ausência de distensão de forma ininterrupta, satura os receptores para a produção de grelina diminuindo, sua produção ${ }^{11}$. No entanto, a redução 
da grelina não é consenso na literatura, alguns autores demonstraram elevação desse peptídio no pós-operatório ${ }^{27}$. A grelina tem efeitos contra-insulínicos através da indução de secreção de hormônios contra-regulatórios como a epinefrina, GH e cortisol ${ }^{11}$. Também diminui a secreção de adiponectina, uma adipocina que promove a sensibilidade à insulina, e diminui a produção de insulina ${ }^{10,11,12}$. Logo, a diminuição de produção de grelina observada no pósoperatório desencadeia outros mecanismos hormonais que favorecem um estado de controle metabólico da diabete.

Vários autores também determinaram aumento da secreção de peptídio YY após bypass gástrico ${ }^{10,33,37,43,42}$. Estudo demonstrou incremento da área abaixo da curva para peptídio YY após a ingestão de pequena refeição nos pacientes operados em relação a dois grupos controle pareados compostos por obesos não operados e por pacientes magros ${ }^{33}$. O nível sérico pós-prandial de peptídio YY sofreu incremento de 10 vezes em relação ao nível basal nos operados; os pacientes dos dois grupos controle apresentaram incremento de 2 a 3 vezes. O PYY pertence à família do polipeptídio pancreático e é produzido por células neuroendócrinas do íleo e do cólon. Promove redução do esvaziamento gástrico, retardo do trânsito intestinal e redução do apetite através da inibição da secreção de neuropeptídio Y no hipotálamo, sendo considerado fator importante de influência na saciedade pós-prandial ${ }^{33}$. Causa ainda diminuição da secreção de grelina, influenciando o controle metabólico da diabete por outro mecanismo do que o da perda de peso ou da redução da ingestão alimentar. Outros peptídios como a neurotensina, a oxintomodulina e o enteroglucagon exercem esse mesmo efeito sobre a grelina. Não existem estudos a respeito da oxintomodulina no pós-operatório de cirurgia bariátrica. Como se trata de peptídio secretado pelas células L do íleo e do cólon espera-se comportamento semelhante ao do GLP-1 ${ }^{12}$. Estudos sobre as alterações dos hormônios do trato gastrointestinal após bypass jejunoileal demonstram elevação do nível sérico de enteroglucagon e neurotensina $a^{31,44}$. Apesar de ser procedimento não realizado atualmente, o bypass jejunoileal representa um modelo em que os nutrientes atingem precocemente o íleo como ocorre na hipótese do intestino posterior. Elevação de enteroglucagon também foi observada após o bypass gástrico e as derivações biliopancreáticas ${ }^{41,59}$.

A leptina é produzida pelo tecido adiposo e afeta diretamente o metabolismo da glicose e dos lipídios. Após a deposição de tecido adiposo, seu nível sérico eleva e exerce ação ao nível do hipotálamo, reduzindo a expressão do orexigênico neuropeptídio Y e elevando a expressão do anorexigênico alfa-melanocítico. Estudos em roedores obesos e diabéticos com ausência de produção de leptina, apresentam melhora da tolerância à glicose e perda de peso após a administração exógena desse peptídio. Em pacientes obesos e diabéticos especula-se que algum fator secretado pelo duodeno e jejuno proximal induza uma forma de resistência à leptina ${ }^{54}$. Como forma de compensação a essa resistência, ocorre elevação dos níveis desse peptídio $^{39}$. O bypass gástrico ou a derivação biliopancreática proporcionam redução precoce dos níveis de leptina, sem correlação com o valor do $\mathrm{IMC}^{26,13}$. Alguns autores sugerem que a derivação do duodeno e jejuno proximal promove diminuição da resistência a leptina e conseqüente redução dos seus níveis séricos ${ }^{56}$.

A adiponectina normalmente apresenta níveis séricos reduzidos em situações de resistência periférica a insulina como na obesidade ${ }^{28,69}$. Ela apresenta relação inversamente proporcional com a insulina sérica ${ }^{69}$. Animais geneticamente modificados que não expressam receptores para a adiponectina apresentam resistência periférica à insulina ${ }^{35}$. A administração exógena de adiponectina em animais melhora a resistência periférica à insulina através da inibição da gluconeogênese, da interferência na produção do fator de necrose tumoral e do aumento da oxidação de ácidos graxos nos músculos ${ }^{18,20,28}$. Estudos demonstram incremento nos níveis de adiponectina após o bypass gástrico. Esse incremento está da dependência da diminuição do IMC, ou seja, é uma alteração hormonal favorável ao controle da diabete no pós-operatório que está na dependência da redução da ingestão de alimentos e da perda de peso. A resistina, o fator de necrose tumoral alfa e a interleucina 6 , são adipocinas que habitualmente estão elevados em obesos e induzem resistência periférica à insulina. A gordura intra-abdominal é a principal fonte de resistina ${ }^{40}$. Os efeitos da cirurgia bariátrica nesses peptídios ainda não são bem estabelecidos.

\section{Novos procedimentos}

Novos procedimentos cirúrgicos têm sido descritos com o objetivo de promover controle da diabete tipo $2^{7,14,58}$. Esses procedimentos utilizam os conhecimentos já adquiridos pelas cirurgias bariátricas tradicionais. Estudos preliminares mostram alguns resultados favoráveis, no entanto esses novos procedimentos ainda são considerados experimentais e devem ser realizados somente através de protocolos de estudos clínicos.

Cohen et al. ${ }^{7}$ recentemente publicaram os resultados de dois pacientes com IMC de $29 \mathrm{~kg} / \mathrm{m}^{2}$ e de $30,3 \mathrm{~kg} / \mathrm{m}^{2}$, com diabete e submetidos ao bypass duodeno-jejunal com seguimento de 12 meses. No primeiro mês após a operação, apresentaram melhora significativa da glicemia de jejum, que se manteve estável até o $9^{\circ}$ mês de pós-operatório. Os níveis de hemoglobina glicosilada normalizaram no $3^{\circ}$ mês até o fim do seguimento e os níveis de insulina sérica reduziram até níveis semelhantes ao de não-diabéticos. Interessante observar que não foi verificada perda de peso nos dois pacientes durante o período de seguimento.

Santoro et al. ${ }^{58}$ publicaram artigo com 55 pacientes submetidos à gastrectomia vertical, omentectomia e ressecção de segmento de intestino delgado com remanescente de 300 $\mathrm{cm}$ (40 cm de jejuno e $260 \mathrm{~cm}$ de íleo $)^{58}$. Na reconstrução intestinal realizaram anastomose jejunoileal a $80 \mathrm{~cm}$ da válvula ileocecal e uma gastroileostomia. Segundo os autores, a vantagem desse procedimento é que evita obstáculos para a ingestão de alimentos, evita segmentos exclusos do trânsito alimentar e não promove disabsorção. A redução média 
do IMC foi de $20,1 \mathrm{~kg} / \mathrm{m}^{2}$ após 12 meses e foi associada a controle de co-morbidades, especialmente a diabete. Houve redução significativa nas dosagens de grelina e de resistina sérica em jejum e aumento nos níveis de GLP-1 e PYY. Os mesmo autores publicaram estudo com 100 pacientes, onde realizaram a gastrectomia vertical associada à ressecção intestinal com remanescente de $300 \mathrm{~cm}$. Na reconstrução intestinal, realizaram somente anastomose entre o jejuno e o íleo. Nesse estudo, dos 34 pacientes com diabetes, 31 apresentaram controle da doença no pós-operatório e três apresentaram melhora do quadro.

De Paula et al. ${ }^{14}$ demonstraram os resultados da interposição ileal associada à gastrectomia vertical (sleeve) por via laparoscópica em 19 pacientes com IMC médio de 40,2 $\mathrm{kg} / \mathrm{m}^{2}$. Desses, cinco tinham diabete tipo 2, três estavam em uso de hipoglicemiantes por via oral e dois de insulina. A gastrectomia vertical foi associada à colocação de banda de silicone 3 a $4 \mathrm{~cm}$ abaixo da cárdia. Foi isolado um segmento de $100 \mathrm{~cm}$ de íleo, localizado a $50 \mathrm{~cm}$ da válvula íleocecal, e interposto de maneira isoperistáltica a $50 \mathrm{~cm}$ do ângulo duodenojejunal. O seguimento médio no pós-operatório foi de 11,6 meses. A perda média de peso foi de $38,4 \%$ nos nove pacientes com seguimento de 12 meses. Após 3 semanas todos os cinco com diabete apresentaram glicemia normal sem o uso de medicações. Após 6 meses os oito pacientes com hipertensão arterial apresentaram normalização da pressão arterial. Todos os pacientes apresentaram níveis normais de colesterol após três semanas; o mesmo para os triglicerídios após 6 meses.

\section{CONCLUSÕES}

Os procedimentos cirúrgicos sobre o trato gastrointestinal, principalmente as cirurgias bariátricas, proporcionam controle metabólico da diabete tipo 2. Entre as cirurgias bariátricas os melhores resultados são obtidos com as derivações biliopancreáticas seguidas pelo bypass gástrico e pela banda gástrica. A resolução da diabete é, em parte, explicada pela perda de peso e diminuição da ingestão de alimentos. Nas derivações biliopancreáticas e no bypass gástrico ocorre ainda alteração na secreção de hormônios, principalmente os de origem no trato digestivo. Duas hipóteses foram formuladas para explicar as modificações desses hormônios: a hipótese do intestino anterior, em que a exclusão do duodeno e do jejuno proximal previne a secreção de algum agente ainda não identificado que promove a resistência periférica à insulina, e a do intestino posterior, em que a derivação intestinal promove a passagem rápida do quimo até o intestino distal e induz a secreção precoce dos hormônios que promovem o controle da diabete. Novos procedimentos cirúrgicos, ainda considerados experimentais, estão demonstrando resultados preliminares favoráveis ao controle metabólico da diabete tipo 2 .

Freitas ACT. Gastrointestinal surgery for the treatment of type 2 diabetes. ABCD Arq Bras Cir Dig 2007; 20(2):119-26.

ABSTRACT - Background - The ability of gastrointestinal surgical interventions, mainly bariatric surgery, to promote the control of type 2 diabetes, has already been well documented. Aim - To review the literature related to the effects of gastrointestinal surgery regarding type 2 diabetes, especially in relation to metabolic control and its physiopathology. Methods - Literature was reviewed on Medline, pages on the internet, references from relevant articles and studies presented and published on the annals of the International Conference on Gastrointestinal Surgery to Treat Type 2 Diabetes, which occurred in Rome in 2007. Conclusions - Among all bariatric surgeries, biliopancreatic diversion, presented the best control rates for type 2 diabetes followed by gastric bypass and gastric banding. This control is related to weight loss and reduction on food intake. Biliopancreatic diversion and gastric bypass also presented important modifications in gut hormones. The most significant ones being: GLP-1, GIP, PYY, ghrelin, leptin, IGF-1, adiponectin. These hormones promote loss of appetite, promote actions over $\beta$ cells, increase the secretion of insulin, and increase insulin sensitivity. Two theories have been formulated to explain the changes observed on these hormones: the foregut theory, where the bypass of the duodenum and proximal jejunum avoids the secretion of an unknown factor that induces insulin resistance; and the hindgut theory, where the early presentation of food to the ileum anticipates the production of hormones that control diabetes. Recently, new promising procedures have been developed. Among them are the duodenal-jejunal bypass, ileal interposition, and intestinal resection associated to vertical gastrectomy. These new procedures are still considered experimental.

HEADINGS - Bariatric surgery. Diabetes mellitus. Biliopancreatic diversion. Gastric bypass. Gastrointestinal hormones.

\section{REFERÊNCIAS}

1. Adami GF, Cordera R, Camerini G, Marinari GM, Scopinaro N. Recovery of insulin sensitivity in obese patients at short term after biliopancreatic diversion. J Surg Res. 2003;113:217-21.

2. Broglio F, Arvat E, Benso A, Gottero C, Muccioli G, Papotti M, van der Lely AJ, Deghenghi R, Ghigo E. Ghrelin, a natural GH secretagogue produced by the stomach, induces hyperglycemia and reduces insulin secretion in humans. J Clin Endocrinol Metab. 2001;86:5083-6.

3. Buchwald H, Avidor Y, Braunwald E, Jensen MD, Pories W, Fahrbach K, Schoelles K. Bariatric surgery: a systematic review and meta-analysis. JAMA. 2004;292:1724-37.

4. Buchwald H, Estok R, Fahrbach K, Banel D, Jensen MD, Pories W, Bantle J, Sledje I. Effects of bariatric surgery on type 2 diabetes: a systematic review and meta-analysis. In: Rubino F, Cummings DE, Kaplan LE, Schauer PR, editors. International conference on gastrointestinal surgery to treat type 2 diabetes: anais - International conference on gastrointestinal surgery to treat type 2 diabetes. Italy: Rome; 2007. p.1-30.
5. Calle EE, Thun MJ, Petrelli JM, Rodriguez C, Heath CW Jr. Body-mass index and mortality in a prospective cohort of U.S. adults. N Engl J Med. 1999;341:1097-105.

6. Cavagham MK, Ehrmann DA, Polonsky KS. Interactions between insulin resistance and insulin secretion in the development of glucose intolerance. J Clin Invest. 2000;106:329-33.

7. Cohen RV, Schiavon CA, Pinheiro JS, Luiz Correa J, Rubino F. Duodenaljejunal bypass for the treatment of type 2 diabetes in patients with BMI 22-34: a report of two cases. Surg Obes Rel Dis. 2007;3:195-7.

8. Cummings DE, Weigle DS, Frayo RS, Breen PA, Ma MK, Dellinger EP, Pummel JP. Plasma ghrelin levels after diet-induced weight loss and gastric bypass surgery. New Engl J Med. 2002;346:1623-30.

9. Cummings DE, Shannon MH. Roles for ghrelin in the regulation of appetite and body weight. Arch Surg. 2003;138:389-96. 
10. Cummings DE, Overduim J, Shannon MH, Foster-Schubert KE. Hormonal mechanisms of weight loss and diabetes resolution after bariatric surgery. Surg Obes Relat Dis. 2005;1:358-68.

11. Cummings DE, Foster-Schubert KE, Overduim J. Ghrelin and energy balance: focus on current controversies. Curr Drug Targets. 2005;6:153-69.

12. Cummings DE, Overduim J, Shannon MH, Foster-Schubert KE. Role of the bypassed proximal intestine in the anti-diabetic effects of bariatric surgery. Surg Obes Relat Dis. 2007;3:109-15.

13. De Marinis L, Mancini A, Valle D, Bianchi A, Milardi D, Proto A, Lanzone A, Tacchino R. Plasma leptin levels alter biliopancreatic diversion: dissociation with body mass index. J Clin Endocrinol Metab. 1999;84:2386-9.

14. De Paula AL, Macedo AL, Prudente AS, Queiroz L, Schraibman V, Pinus J. Laparoscopic sleeve gastrectomy with ileal interposition ("neuroendocrine break") - pilot study of a new operation. Surg Obes Relat Dis. 2006;2:4647.

15. Druker DJ. Glucagon-like peptide-1 and the islet beta-cell: augmentation of cell proliferation and inhibition of apoptosis. Endocrinology. 2003;144:5145-8.

16. Druker DJ. The role of gut hormones in glucose homeostasis. J Clin Invest. 2007;117:24-32.

17. Faraj M, Havel PJ, Phelis S, Blank D, Sniderman AD, Cianflone K. Plasma acylation-stimulating protein, adiponectin, leptin, and ghrelin before and after weight loss induced by gastric bypass surgery in morbidly obese subjects. J Clin Endocrinol Metab. 2003;88:1594-602.

18. Fasshauer M, Klein J, Neumann S, Eszlinger M, Paschke R. Hormonal regulation of adiponectin gene expression in 3T3-L1 adipocytes. Biochem Biophys Res Commun. 2002;190:1084-9.

19. Friedman JE, Dohm GL, Leggett-Frazier N, Elton CW, Tapscott EB, Pories WP, Caro JF. Restoration of insulin responsiveness in skeletal muscle of morbidly obese patients after weight loss: effect on muscle glucose transport and glucose transporter GLUT4. J Clin Invest. 1992;89:701-5.

20. Fruebis J, Tsao TS, Javorschi S, Ebbets-Reed D, Erickson MR, Yen FT, Bihain $\mathrm{BE}$, Lodish HF. Proteolytic cleavage product of 30-kDa adipocyte complement-related protein increases fatty acid oxidation in muscle and causes weight loss in mice. Proc Natl Acad Sci USA. 2001;98:2005-10.

21. Fruhbeck G, Caballero AD, Gil MJ. Fundus functionality and ghrelin concentrations after bariatric surgery. New Engl J Med. 2004;350:308-9.

22. Geloneze B, Tambascia MA, Pilla VF, Geloneze SR, Repetto EM, Pareja JC. Ghrelin, a gut-brain hormone: effect of gastric bypass surgery. Obes Surg. 2003:13:17-22.

23. Gray RE, Tanner CJ, Pories WJ, MacDonald KG, Houmard JA. Effect of weight loss on muscle lipid content in morbidly obese subjects. Am J Physyol Endocrinol Metab. 2003;284:e726-e32.

24. Greenway SE, Greenway FL, Klein S. Effects of obesity surgery on noninsulin-dependent diabetes mellitus. Arch Surg. 2002;137:1109-17.

25. Halverson JD, Kramer J, Cave A, Permutt A, Santiago J. Altered glucose tolerance, insulin response, and insulin sensitivity after massive weight reduction subsequent to gastric bypass. Surgery. 1982;92:235-40.

26. Hickey MS, Pories WJ, MacDonald KG, Cory KA, Dohm GL, Swanson MS, Israel RG, Barakat HA, Considine RV, Caro JF, Houmard JA. A new paradigm for type 2 diabetes mellitus: could it be a disease of the foregut? Ann Surg. 1998;227:637-44.

27. Holdstock C, Engstrom BE, Öhrvall M, Lind L, Sundbom M, Karlsson FA. Ghrelin and adipose tissue regulatory peptides: effect of gastric bypass surgery in obese humans. J Clin Endocrinol Metab. 2003;88:3177-83.

28. Hotta K, Funahashi T, Arita Y, Takahashi M, Matsuda M, Okamoto Y, Iwahashi H, Kuriyama H, Ouchi N, Maeda K, Nishida M, Kihara S, Sakai N, Nakajima T, Hasegawa K, Muraguchi M, Ohmoto Y, Nakamura T, Yamashita S, Hanafusa T, Matsuzawa Y. Plasma concentrations of a novel, adipose-specific protein, adiponectin, in type 2 diabetic patients. Arterioscler Thromb Vasc Biol. 2000;20:1595-99.

29. Houmard JA, Tanner CJ, Yu C, Cunningham PG, Pories WJ, MacDonald KG, Shulman GI. Effect of weight loss on insulin sensitivity and intramuscular longchain fatty acyl-CoAs in morbidly obese subjects. Diabetes. 2002;51:295963.

30. Brasil. IBGE [online]. Brasília: Ministério do Orçamento, Planejamento e Gestão. [acesso em 27 maio 2007]. Pesquisa de orçamentos familiares - POF 2002-2003. Disponível em: http:/www.ibge.gov.br/home/estatistica/populacao/condicaodevida/pof/2002/default.shtm.

31. Kellum JM, Kuemmerle JF, O'Dorisio TM, Rayford P, Martin D, Engle K, Wolf L, Sugerman HJ. Gastrointestinal hormone responses to meals before and after gastric bypass and vertical banded gastroplasty. Ann Surg. 1990;211:763-70.

32. Koopsmans HS, Ferri GL, Sarson DL, Polak JM, Bloom SR. The effects of ileal transposition and jejunoileal bypass on food intake and GI hormone levels in rats. Physiol Behav. 1984;33:601-9.

33. Korner J, Bessler M, Cirilo LJ, Conwell IM, Daud A, Restuccia NL, Wardlaw SL. Effects of Roux-en-Y gastric bypass surgery on fasting postprandial concentrations of plasma ghrelin, peptide YY, and insulin. J Clin Endocrinol Metab 2005;90:359-65.

34. Koro CE, Bowlin SJ, Bourgeois N, Feder DO. Glycemic control from 1988 to 2000 among US adults diagnosed with type 2 diabetes: a preliminary report. Diabetes Care. 2004;27:17-20.
35. Kubota N, Terauchi Y, Yamauchi T, Kubota T, Moroi M, Matsui J, Eto K, Yamashita T, Kamon J, Satoh H, Yano W, Froguel P, Nagai R, Kimura S, Kadowaki T, Noda T. Disruption of adiponectin causes insulin resistance and neointimal formation. J Biol Chem. 2002;277:25863-6.

36. Le Roith D. Insulin-like growth factors. N Engl J Med. 1997;336:633-40.

37. Le Roux CW, Aylwin SJ, Batterham RL, Borg CM, Coyle F, Prasad V, Shurey S, Ghatei MA, Patel AG, Bloom SR. Gut hormones profiles following bariatric surgery favor an anoretic state, facilitate weight loss, and improves metabolic parameters. Ann Surg. 2006;243:108-14.

38. Lynn FC, Pamir N, NG EH, McIntosh CH, Kieffer TJ, Pederson RA. Defective glucose-dependent insulinotropic polypeptide receptor expression in diabetic fatty Zucker rats. Diabetes. 2001;50:1004-11.

39. Mantzoros CS. The role of leptin in human obesity and disease: a review of current evidence. Ann Intern Med. 1999;20:671-80.

40. Mc Ternan CL, Mc Ternan PG, Harte AL, Levick PL, Barnett AH, Kumar S. Resistin, central obesity and type 2 diabetes. Lancet. 2002;359:46-7.

41. Meryn S, Stein D, Straus EW. Pancreatic polypeptide, pancreatic glucagons, and enteroglucagon in morbid obesity and following gastric bypass operation. Int J Obes. 1986;10:37-42.

42. Morinigo R, Lacy AM, Casamitjana R, Delgado S, Gomis, R, Vidal J. GLP-1 and changes in glucose tolerance following gastric bypass surgery in morbidly obese subjects. Obes Surg. 2006;16:1594-601.

43. Morinigo R, Moize V, Musri M, Lacy AM, Navarro S, Marin JL, Delgado S, Casamitjana R, Vidal J. Glucagon-like peptide-1, peptide YY, hunger, and satiety after gastric bypass surgery in morbidly obese subjects. J Clin Endocrinol Metab. 2006;91:1735-40.

44. Naslund E, Backman L, Holst JJ, Theodorsson E, Hellstrom PM. Importance of small bowel peptides for the improved glucose metabolism 20 years after jejunoileal bypass for obesity. Obes Surg. 1998;8:253-60.

45. National institute of diabetes and digestive and kidney diseases [online]. Bethesda: National institutes of health; 2007 [cited 2007 may 18]. Complications of diabetes in the United States. Available from: http//diabetes.niddk.nih. gov/dm/pubs/statistics/index.htm\#13.

46. Nauck MA, Heimesaat MM, Orskov C, Holst JJ, Ebert R, Creutzfeldt W. Preserved incretin activity of glucagon-like peptide 1 [7-36 amide] but not of syntetic human gastric inhibitory polypeptide in patients with type 2 diabetes mellitus. J Clin Invest. 1993;91:301-7.

47. Patriti A, Facchiano E, Annetti C, Aisa MC, Galli F, Fanelli C, Donini A. Early improvement of glucose tolerance after ileal transposition in a non-obese type 2 diabetes rat model. Obes Surg. 2005; 15:1258-64.

48. Pender C, Goldfine ID, Tanner CJ, Pories WJ, MacDonald KG, Havel PJ, Houmard JA, Youngren JF. Muscle insulin receptor concentrations in obese patients post bariatric surgery: relationship to hyperinsulinemia. Int J Obes. 2004;28:363-9.

49. Pories WJ, MacDonald KG, Morgan EJ, Sinha MK, Dohm GL, Swanson MS, Barakat HA, Khazanie PG, Legget-Frazier N, Long SD. Surgical treatment of obesity and its effect on diabetes. Am J Clin Nutr. 1992;55:582s-5s.

50. Pories WJ, Swanson MS, MacDonald KG, Long SB, Morris PG, Brown BM, Barakat HA, de Ramon RA, Israel G, Dolezal JM. Who would have thought it? An operation proves to be the most effective therapy for adult-onset diabetes mellitus. Ann Surg. 1995;222:339-50.

51. Pories WJ, Albrecht RJ. Etiology of type II diabetes mellitus: role of the foregut. World J Surg. 2001;25:527-31.

52. Pories WJ. Diabetes: the evolution of a new paradigm. Ann Surg. 2004;239:123.

53. Poulos JE, Legget-Frazier N, Khazanie P, Long S, Sportsman R, MacDonald $\mathrm{K}$, Caro JF. Circulating insulin-like growth factor I concentrations in clinically severe obese patients with and without NIDDM in response to weight loss. Horm Metab Res. 1994;26:478-80.

54. Rubino F, Gagner M. Potencial of surgery for curing type 2 diabetes mellitus. Ann Surg. 2002;236:554-9.

55. Rubino F, Marescaux J. Effect of duodenal exclusion in a non-obese animal model of type 2 diabetes. Ann Surg. 2004:239:1-11.

56. Rubino F, Gagner M, Gentileschi P, Kini S, Fukuyama S, Feng J, Diamond E. The early effect of the Roux-en-Y gastric bypass on hormones involved in body weight regulation and glucose metabolism. Ann Surg. 2004;240:236-42.

57. Rubino F, Forgione A, Cummings DE, Vix M, Gnuli D, Mingrone G, Castagneto M, Marescaux J. The mechanism of diabetes control after gastrointestinal bypass surgery reveals a role of the proximal small intestine in the pathophysiology of type 2 diabetes. Ann Surg. 2006;244:741-9.

58. Santoro S, Malzoni CE, Velhote MC, Milleo FQ, Santo MA, Klajner S, Damiani D, Maksoud JG. Digestive adaptation with intestinal reserve: a neuroendocrinebased operation for morbid obesity. Obes Surg. 2006;16:1371-9.

59. Sarson DL, Scopinaro N, Bloom SR. Gut hormone changes after jejunoileal (JIB) or biliopancreatic (BPD) bypass surgery for morbid obesity. Int J Obes. 1981;5:471-80

60. Schauer PR, Burguera B, Ikramuddin S, Sayeed MD, Cottam D, Gourash W, Hamad G, Eid G, Mattar S, Ramanathan R, Barinas-Mitchel E, Harsha RR, Kuller L, Kelley D. Effect of laparoscopic Roux-em-Y gastric bypass on type 2 diabetes mellitus. Ann Surg. 2003;238:467-85.

61. Scopinaro N, Adami GF, Marinari GM, Gianetta E, Traverso E, Friedman D, Camerini G, Baschieri G, Simonelli A. Biliopancreatic diversion. World J Surg. 1998;22:936-46. 
62. Sieree R, Shaw J, Zimmet P. The global burden of diabetes. In: Gan D, editor Diabetes Atlas. Bruxelas: International Diabetes Federation; 2003. p.15-71.

63. Simpson HL, Umpleby AM, Russel-Jones DL. Insulin-like growth factor-I and diabetes. A review. Growth Horm IGF Res. 1998;8:83-95.

64. Sjöström L, Lindroos AK, Peltonen M, Togerson J, Bouchard C, Carlsson B, Dahlgren S, Larson B, Narbro K, Sjoström CD, Sullivan M, Wedel H. Lifestyle, diabetes, and cardiovascular risk factors 10 years after bariatric surgery. N Engl J Med. 2004;351:2683-93.

65. Strader AD, Vahl TP, Jandacek RJ, Woods SC, D`Alessio DA, Seeley RJ. Weight loss through ileal transposition is accompanied by increased ileal hormone secretion and synthesis in rats. Am J Physiol Endocrinol Metab. 2005;288:e447-e53.

66. Wren AM, Seal LJ, Cohen MA, Brynes AE, Frost GS, Murphy KG, Dhillo WS, Ghatei MA, Bloom SR. Ghrelin enhances appetite and increases food intake in humans. J Clin Endocrinol Metab. 2001;86:5992.

67. Valverde I, Puente J, Martin-Duce A, Molina L, Lozano O, Sancho V, Malaisse WJ, Villanueva-Penacarrillo ML. Changes in GLP-1 secretion after biliopancreatic diversion or vertical banded gastroplasty in obese subjects. Obes Surg. 2005; $15: 387-97$
68. Yde $\mathrm{H}$. The growth hormone dependent sulfation factor in serum from patients with various types of diabetes. Acta Med Scand. 1969;186:293-7.

69. Yang W, Lee W, Funahashi T, Tanaka S, Matsuzawa Y, Chao C, Chen C, Tai T, Chuang L. Weight reduction increases plasma levels of an adipose derived anti-inflammatory protein, adiponectin. J Clin Endocrinol Metab. 2001;86:3815-9.

70. Zander M, Madsbad S, Madsen JL, Holst JJ. Effect of 6-week course of glucagons-like peptide 1 on glycaemic control, insulin sensitivity and beta cell function in type 2 diabetes: a parallel-group study. Lancet. 2002;359:82430 .

71. Zimet P, Alberti KG, Shaw J. Global and societal implications of the diabetes epidemic. Nature. 2001;414:782-7.

Conflito de interesse: não há Fonte financiadora: não há Recebido para publicação em: 15/01/2007 Aceito para publicação em: 22/04/2007 